\title{
Primary immunodeficiencies
}

\section{Teresa Espanol}

Immunology Unit, University Hospital Vall d'Hebron, Barcelona, Spain

E-mail: tespanol@vhebron.net

\section{Introduction}

Primary immunodeficiencies (PID) are inherited disorders of immune system function $[1,2]$ characterised by absent or low response to infectious agents and a high prevalence of autoimmune manifestations and lymphoproliferative diseases.

More than 120 PID in which a molecular defect causes the disease have been identified. The IUIS/WHO Experts Committee published a classification of all PID known to date [3]. A large group are diagnosed in childhood. The incidence of PID is not very high, except for IgA deficiency (IgA-D), which is mostly asymptomatic [4], and occurs in approximately $1 / 600$ of the Caucasian population. However, many PID may be underdiagnosed and therefore, the real incidence of PID might be underestimated [5].

A simplified version of the IUIS classification [3] could be:

A) Antibody production deficiencies that include X-linked Agammaglobulinaemia (XLA), Hyper-IgM, CVID, etc.

B) Combined ID with different degrees of T- and B-cell defects that include all variants of Severe Combined ID (SCID), Wiskott- Aldrich syndrome, etc.

C) Innate immune response defects, such as the phagocytic cell defects in chronic granulomatous disease (CGD), IL-12/IFN $\gamma$ pathway defects, complement deficiencies, etc.

D) A group of well-defined syndromes with defects in apoptosis, DNA repair, etc.

Therapy differs for cases in each group.

A) Antibody production deficiencies are the most frequent group of PID and are characterised by repeated bacterial infections of the respiratory and gastrointestinal tract with increased autoimmunity disorders [6].

Diagnosis is often delayed [7] owing to the great variability in clinical manifestations. Early diagnosis and therapy are essential for the best prognosis and quality of life in these patients. In some of these PID, such as XLA, the molecular defect causing disease is known, which allows a very specific diagnosis of the patient and carriers and, thus, genetic counselling [8]. 
Common variable immunodeficiency (CVID) is the second most frequent PID and can present at any age, in children and young adults, with an autosomal recessive or dominant pattern or as sporadic cases. Diagnosis of probable CVID is based on a marked decrease (at least 2 SD below the mean for age) in two out of three of the major isotypes ( $\operatorname{IgM}, \operatorname{IgG}$ and $\operatorname{IgA}$ ) in patients over the age of 2 years with absent isohaemagglutinins and/or poor response to vaccines, as defined by ESID (www.esid.org) and others. Other defined causes of hypogammaglobulinaemia must be ruled out.

Bronchiectasis and malabsorption, which might be present when the diagnosis is made and therapy is initiated, render complete recovery from sequelae very difficult and prognosis uncertain. Outcome in most cases, despite replacement therapy, is variable and reliable markers for prognosis are lacking.

No definitive molecular cause of CVID, as in IgA-D, has been described, although some genetic defects have recently been implicated in some familial and sporadic cases.

B) Combined immunodeficiencies are the most severe forms of PID. They present with viral and opportunistic infections, usually in the early months or years of life, which are incompatible with life [9] if no proper therapy is applied. This is the case of all forms of SCID in which a haematopoietic precursor transplant (HPT) is compulsory before 1year of age. The degree of T- and B-cell abnormalities influences the therapeutic approach. Most of these PID witth a molecular defect have been identified, facilitating genetic counselling.

C) Defects of phagocytic cells: in the number, such as congenital neutropenias, or defects of their function such as chronic granulomatous disease (CGD), characterised by a defective oxidative burst (due to the absence or malfunction of NADPH oxidase subunits) leading to high susceptibility to infections by catalase-positive bacteria and intracellular organisms. Patients present most frequently with recurrent infections and abscesses in lymph nodes, lung and liver, but also in bones (osteomyelitis), and septicaemia in some cases.

Defects in the IL-12/IFN $\gamma$ pathway are characterised by infections with micobacteria that usually respond to conventional antibiotic therapies. Severity is variable depending on whether they have a complete or partial defect.

Other even more rare defects include abnormalities of the TLR- pathways. These patients have very mild inflammatory manifestations ( mainly no fever when severe infections are diagnosed). And most probably new ID will be defined in the future.

D) Other well-defined immunodeficiencies:

There is a wide range of diseases that have clear-cut phenotypic characteristics (Di George Syndrome, Ataxia-telangiectasia, Wiskott-Aldrich syndrome, etc.) with associations with haematological, neurological and cardiac manifestations and a different spectrum of T, B and NK defects impossible to describe in a short review. There are excellently defined in the books cited and in many papers [10]. 


\section{PID therapies}

\subsection{Gammaglobulin replacement therapy}

Following the description by Dr Bruton of the benefit of gammaglobulin therapy in a boy with agammaglobulinaemia, therapy with intramuscular Ig (IMIg) was the standard treatment up to the beginning of the 1980's. The low levels obtained (there is a local proteolysis) with painful administration currently limit its use.

\subsubsection{Intravenous immunoglobulins (IVIG)}

IVIG is the most widely used treatment for PID [11,12] and is indicated in all forms of antibody deficiencies ( XLA, CVID, Hyper-IgM, etc.) and in combined immunodeficiency prior to and during haematopoietic precursor transplant (HPT).

IVIG has significant advantages, including easy administration of large doses, with good tolerance. Furthermore, all the available preparations approved by the FDA and EMEA have a half-life of 18 to 25 days, contain all IgG subclasses, have minimal anticomplement activity, have a broad spectrum of antibodies and are free of hepatitis B, $\mathrm{C}$, HIV and other viruses. The recent implementation of virus inactivation methods renders these products, which are available in our setting, very safe.

The recommended dose to avoid infections or hospitalisations and improve lung function is 400 to $600 \mathrm{mg} / \mathrm{kg}$ / every 3 weeks, in order to maintain IgG levels above $600 \mathrm{mg} / \mathrm{dl}$, just prior to a new administration.

The adverse effects of IVIG infusion of Ig such as headache, nausea, vomiting, joint pain and/or abdominal pain can occur in $5-15 \%$ of patients and can be minimised by slowing the infusion rate and with pre-treatment with oral paracetamol or antihistamines.

\subsubsection{Subcutaneous immunoglobulins (SCIG)}

SCIG, used for years in the north of Europe, are an alternative to IVIG and permit at-home therapy [13].

As the technique for inserting a small perfusion needle subcutaneously is simple, the medication can be self-administered into the abdominal wall or thighs. The injections are well tolerated. Local reactions are minimal and include erythema and/or pain; systemic reactions are rare.

The monthly dose used is the same but is divided over four weeks. The SCIgG concentration is $16 \%$ to be infused with the aid of a small, battery-powered perfusion pump.

The standard doses used are 100 to $150 \mathrm{mg} / \mathrm{kg}$ / week, corresponding to a dose of 45-60 $\mathrm{ml}$ of a16\% solution. Comparative studies on the efficacy and safety of IVIG and SCIG for replacement therapy found no significant differences with respect to the number of infections or adverse reactions, and recent studies have shown that SCIG administered at home is associated with better quality of life than IVIG administered 
at the hospital; patients and their families enjoy greater independence and greater control over aspects of treatment and their daily life.

Patients with deficiencies of IgG subclasses with IgA deficiency and/or anti-IgA antibodies are at risk of adverse reactions.

\subsection{Haematopoietic precursor transplant}

Haematopoietic precursor transplant (HPT) (14) in PID is aimed at restoring the number and/or function of lymphocytes or phagocytes. It is indicated in PID of $\mathrm{T}$ lymphocytes, combined $\mathrm{T}$ and $\mathrm{B}$ defects and phagocyte defects, which would otherwise almost certainly be fatal. For all different types of SCID, it is the only therapy that can "cure" the disease. In such cases, the diagnosis is urgent to avoid infections that condition their outcome.

\subsubsection{Donor selection}

Syngenic identical related (homozygotic twin) or HLA-compatible allogeneic donors (siblings) are the mostly common used. Matched unrelated allogeneic donors, such as BM donors and cord blood, are also used when siblings are not available.

For HPT in PID, haploidentical donors (parents) are rarely used owing to the high risk of severe GVHD. The choice is based on availability, condition of the patient and urgency, as well as on the underlying disease.

\subsubsection{Sources of Haematopoietic precursor $(H P)$ cells}

1. Bone marrow.

2. Cord blood: this is a rich source of CD34 + CD38- precursor cells [15] and these cells usually cause less GVHD.

3. Peripheral blood (mobilisation of CD34 + precursor cells to the periphery using G-CSF, with leukapheresis.

\subsubsection{Graft-versus-host-disease (GVHD) [16]}

This is due to recognition of the host tissues by the donor T lymphocytes ("reverse rejection"). If an HLA Class II mismatch exists, the disorder is usually very severe or even fatal. The minor histocompatibility antigens give rise to a greater or lesser degree of GVHD despite matching (even if "complete") in the majority of patients transplanted for PID. It is more common and/or intense with unrelated donors. The results are better the earlier HPT is performed, in the absence of previous infections and with the optimum degree of matching.

\subsection{Innate immune response defect therapy}

This group of PIDs includes a very wide range of immunological abnormalities with different therapies. Severe cases can also be treated with HPT but, as for CGD, a defect in phagocytic function, only with a good compatible donor (sibling) owing 
to the risk of GVHD. In the majority of these patients, prophylactic antibiotics and antifungal drugs [17] are the standard therapeutic protocol. The use of cytokines, such as IFN $\gamma$ [18], is also well accepted in CGD and some cases of IL12/IFN $\gamma$ pathway defects. Conventional antibiotics and anti-micobacterial drugs are usually effective in this latter group of patients.

Most probably, new therapeutic protocols will be developed for innate immune response defects (use of IFN $\alpha / \beta$, stimulators or inhibitors of TOL-like receptors, etc.) as new types of PID are described.

\subsection{Other therapies}

Antiinflammatories, or even immunosuppressors, have to be used in specific circumstances such as autoimmune manifestations, malabsorption, lymphoid infiltrates, etc.

Nutrition protocols and physiotherapy are to be taken into account, together with psycological and social support.

\subsection{Gene therapy [19]}

PID are ideal candidates for this therapy in the near future. They are monogenic, haematopoietic cells are easily obtained and virus replication is easy within them. Vectors (viruses) "infect" the stem cells of the patient's bone marrow, producing transfection of the wild (healthy) gene in these cells. Encouraging results have been achieved in X-linked SCID as there are a number of patients considered "cured". However, some neoplastic processes have occurred due to the activation of protooncogenes close to the point of insertion of the external gene. Trials with adenovirus, physical methods (direct injection...) and chemical methods (viral modification, artificial viruses... ) are currently underway.

\subsection{In conclusion}

PID diagnosis and therapy have changed greatly in the last 15-20 years. Molecular diagnosis has allowed better definition of many diseases, and permit prenatal diagnosis, genetic counselling, etc. Further advances can be expected in coming years [20] which will probably improve even more the diagnosis and prognosis of PID patients.

\section{Key challenges for patients with PID worldwide}

The main problem facing many patients is to be diagnosed correctly in order to receive a propriate therapy (gammaglobulin replacement therapy, HPT or any therapy related to each case) and to be diagnosed in time before sequelae, such as bronchiectasis, severe opportunistic infections, malabsorption, etc are established. 
Most PID are rare diseases and not many physicians are aware of them, or know that a child or an adult (even less aware!) patient can have a primary immunodeficiency. If the diseases are not suspected, no appropriate study will be performed and diagnosis established.

\section{Opportunities for patients with PID}

Most PID have a specific therapy which can, in many cases, offer a near-to-normal life. However, these therapies must be introduced as soon a s possible, before sequelae of the repeated and severe infections are present. The combined forms of PID (such as SCID) need to be isolated in a laminar flow room to avoid new infections, and an HPT sought, since it is the only therapeutic measure available to save their lives. All hypogammaglobulinaemic patients must receive continuous therapy with gammaglobulin infusions that will combat the existing infections and prevent new ones.

PID have been excellent models of how the immune response works and their diagnosis and the wide research carried out in the last 20 years has led to better therapies for them and many other immunological diseases.

\section{Examples of physician initiatives internationally}

European Society for Immunodeficiencies (ESID): www.esid.org Latinamerica Society for Immunodeficiencies (LASID): www.Lasid2009.org

Information about diagnosis and therapy can also be found at these different webs: American Academy of Asthma, Allergy, and Immunology: http://www.aaaai.org/ Immune Deficiency Foundation: http://www.primaryimmune.org/

Jeffrey Modell Foundation: www.jmfworld.com

International Patients Organization for Primary Immunodeficiencies ( IPOPI):

www.ipopi.org/.

\section{References}

[1] Primary immunodeficiency diseases, in: A Molecular and Genetic Approach, (2nd ed.), H.D. Ochs, C.I.E. Smith and J.M. Puck, eds, Oxford University Press, New York, 2007.

[2] Primary Immunodeficiency Diseases, in: Definition, Diagnosis and Management, N. Rezaei, A. Aghamohammadi and L.D. Notarangelo, eds, Springer-Verlag Berlin, 2008.

[3] R.S. Geha, L.D. Notarangelo, J.L. Casanova, H. Chapel, M.E. Conley, A. Fischer, L. Hammarström et al., International Union of Immunological Societies Primary Immunodeficiency Diseases Classification Committee. Primary immunodeficiency diseases: an update from the International Union of Immunological Societies Primary Immunodeficiency Diseases Classification Committee, J Allergy Clin Immunol 120(4) (Oct 2007), 776-794. 
[4] A.H. Latiff and M.A. Kerr, The clinical significance of immunoglobulin A deficiency, Ann Clin Biochem 44 (2007), 131-139.

[5] J.M. Boyle and R.H. Buckley, Population prevalence of Diagnosed primary Immunodeficiency diseases in the united States, J Clin Immunol 27 (2007), 497-502.

[6] A.K. Knight and C. Cunningham-Rundles, Inflammatory and autoimmune complications of common variable immune deficiency. Autoimmun Rev 5 (2006), 156-159.

[7] B. Seymour, J. Miles and M. Haeney, Primary antibody deficiency and diagnostic delay, J Clin Pathol 58 (2005), 546-547.

[8] E. Rantanen, M. Hietala, U. Kristoffersson et al., Regulation and practices of genetic counselling in 38 European countries: the prespective of national representatives, European J Human Genetics 16 (2008), 1208-1216.

[9] R.H. Buckley, The multiple causes of human SCID, J Clin Invest (2004), 1409-1411.

[10] F.A. Bonilla, I.L. Bernstein, D.A. Khan, Z.K. Ballas, J. Chinen, M.M. Frank, L.J. Kobrynski, A.I. Levinson, B. Mazer, R.P. Nelson, Jr., J.S. Orange, J.M. Routes, W.T. Shearer and R.U. Sorensen, American Academy of Allergy, Asthma and Immunology; American College of Allergy, Asthma and Immunology; Joint Council of Allergy, Asthma and Immunology. Practice parameter for the diagnosis and management of primary immunodeficiency, Ann Allergy Asthma Immunol 94(5 Suppl 1) (May 2005), S1-S63.

[11] P. Wood, S. Stanworth, J. Burtonn, A, Jones, D.G. Peckham, T. Green, C. Hyde and H. Chapel, Recognition, clinical diagnosis and management of patients with primary antibody deficiencies: a systematic review, Clin Exp Immunol 149 (2007), 410-423.

[12] J.M. García, T. Español, M.D. Gurbindo and C. Casas, Update on the treatment of primary immunodeficiencies, Allergol Immunopathol (Madr) 35(5) (Sep 2007), 184-192.

[13] A. Gardulf, U. Nicolay, O. Asensio, E. Bernatowska et al., Children and adults with primary antibody deficiencies gain quality of life by subcutaneous IgG self-infusions at home, J Allergy Clin Immunol 114 (2004), 936-942.

[14] L.D. Notarangelo, C. Forino and E. Mazzolari, Stem cell transplantation in primary immunodeficiencies, Curr Op Allergy Clin Immunol 6 (2006), 443-448.

[15] J.J. Ortega, J. Yaniv, V. Rocha et al., on behalf of Eurocord-CBTG. Cord blood transplant for inborn errors, Blood 92(Sup 1) (1998), 291.

[16] G.E. Sale, Pathogenesis of graft-versus-host-disease, Biol Blood Marrow Transplant 11 (2005), 21-23.

[17] R.A. Seger, Modern management of chronic granulomatous disease, Haematol 140 (2008), 255266.

[18] B.E. Marciano, R. Wesley, E.S. De Carlo et al., Long-term interferon-gamma therapy for patients with chronic granulomatous disease, Clin Infect Dis 39 (2004), 692-699.

[19] M. Cavazzana-Calvo and A. Fischer, Gene therapy for severe combined immunodeficiency: are we there yet, J Clin Invest 117 (2007), 1456-1467.

[20] J.L. Casanova, Abel L Primary Immunodeficiencies, A field in its infancy, Science 317 (2007), $617-619$. 